\title{
Synthesis of Guerbet Amine-Oxide for Enhanced Oil Recovery
}

\author{
Changxin Shi ${ }^{1},{ }^{1,2}$ Zhiping Li, ${ }^{1}$ Hong Zhang, ${ }^{2}$ Yajuan Chen, ${ }^{2}$ Minglong Zhang, \\ and Meng $\mathrm{He}^{2}$ \\ ${ }^{1}$ China University of Geosciences, Beijing 100083, China \\ ${ }^{2}$ Research Institute of Exploration and Development, PetroChina Huabei Oilfield Company, Renqiu 062550, China
}

Correspondence should be addressed to Changxin Shi; cxshi@qq.com

Received 17 October 2019; Revised 9 January 2020; Accepted 24 January 2020; Published 19 February 2020

Academic Editor: Patricia E. Allegretti

Copyright ( 2020 Changxin Shi et al. This is an open access article distributed under the Creative Commons Attribution License, which permits unrestricted use, distribution, and reproduction in any medium, provided the original work is properly cited.

Guerbet amine-oxide was synthesized by using Guerbet acid, $N, N^{\prime}$-diethyl-1,3-propanediamine and hydrogen peroxide. Its structure was confirmed by FT-IR spectra and mass spectra. The critical micelle concentration (CMC) and the interfacial tension between the crude oil and water were measured. The results showed that the $\mathrm{CMC}$ of the Guerbet amine-oxide is $2.14 \times 10^{-5} \mathrm{~mol} / \mathrm{L}$ and the interfacial tension could be lowered to ultralow levels. The core flood test showed that the surfactant and polymer binary system could increase oil recovery by $24.7 \%$ above the water flood.

\section{Introduction}

China's oil import dependence has been rising since 1993 and will exceed $70 \%$ in 2020 [1]. Meanwhile, the average recovery factor from China's oilfields is only $24.11 \%$. Clearly, it is needed to enhance oil recovery in China. In the past few years, in order to improve oil recovery, alkaline-surfactant-polymer (ASP) filed tests have been carried out in China's Daqing oil field and showed great success [2]. The use of alkali can reduce surfactant adsorption on reservoir rock and generate in situ surfactants with acidic oils, and these in situ generated surfactants can produce synergistic effects with the added surfactants in removing residual oil [3]. However, it also caused some side effects, including scaling and corrosion damage to the lifting system, strong emulsification, and greatly decreased liquid production [4]. For these reasons, some surfactant-polymer pilot tests have been performed in China [5]. Undoubtedly, surfactants play a very important role in the flooding formulations. According to capillary number theory, to mobilize trapped residual oil, it often requires a low interfacial tension to overcome capillary forces to allow the oil to flow [6]. The application of surfactants can effectively reduce the interfacial tension; thus, it becomes a major parameter for evaluating the performance of flood surfactants. Generally speaking, the tighter the surfactant arranges at the oil/water interface, the lower interfacial tension can be obtained [7]. The hydrophilicity of the branched surfactant head groups can overcome the steric repulsion between the tails and forms a compact layer, which has a positive effect on reducing the interfacial tension at the oil/water interface $[8,9]$. Guerbet alcohol is a type of branched alcohol, which is substituted by an alkyl group in the 2-position. Some literatures have reported its use as a raw material for the preparation of oil displacement gents [10, 11]. However, some of these surfactants are poor hydrolytic stability [12], or their synthetic routes are relatively complicated.

Amine-oxide surfactants are a class of zwitterionic surfactants [13]. They exhibit cationic behaviour in acid solution [14] and characteristics of a nonionic surfactant in an alkaline condition [15]. These surfactants show less irritation to the skin, high foaming properties, and excellent thickening function [16], which is benefit for oil recovery [17].

In this work, we synthesized the Guerbet amine-oxide and investigated its performance in oil recovery.

\section{Experimental}

2.1. Materials. Guerbet acid (2-decyltetradecanoic acid) (commercial product) was purchased from Jin Tenglong Trade Co., Ltd. N,N-dimethyl-1,3-propane diamine (analytically grade) was purchased from Tci Company, and 
phosphorus trichloride was obtained from West Asia Reagent Company. Hydrogen peroxide (30\%) was purchased from Aladdin Reagent Company. Ethylenediaminetetraacetic acid disodium salt was purchased from Tianjin Damao Reagent Company. Dehydrated crude oil with a density of $810 \mathrm{~kg} / \mathrm{m}^{3}$ and a viscosity of $9 \mathrm{MPa} \cdot \mathrm{s}$ was derived from the 733 well of the Jing 11 block. Partially hydrolyzed polyacrylamide (HPAM) with an average molecular weight of $17 \mathrm{MDa}$ and a degree of hydrolysis of $23 \%$ was provided by Hengju Company with the commercial name of KY-4s.

The water used in the experiments is formation water with a salinity of $10951 \mathrm{mg} / \mathrm{L}$.

2.2. Analytical Methods. The FT-IR spectra were recorded using a Thermo Nicolet is5 recording spectrometer.

ESI-MS spectra were obtained on a WatersXevoTQ mass spectrometer. The instrument was operated in the positive ion mode.

Measurements of the surface tension were carried out on a Junao tensiometer.

The Interfacial tensions were measured by means of the spinning-drop technique at $54^{\circ} \mathrm{C}$ using a TX 550 spinning-drop interfacial tensiometer at the rotational speed of $5000 \mathrm{rpm}$.

Viscosity measurements were conducted on a Haake Mars rheometer at $54^{\circ} \mathrm{C}$ with a shear rate of $7.34 \mathrm{~s}^{-1}$.

2.3. Synthesis. The synthetic route of the Guerbet amineoxide is illustrated in Scheme 1.

2.3.1. Synthesis of 2-Decyltetradecanoyl Chloride. In a $100 \mathrm{ml}$ three-neck flask equipped with a magnetic stirrer, $14.72 \mathrm{~g}$ of 2-decyltetradecanoic acid was added, and the flask was placed into an oil bath in a controlled temperature oil bath at $85^{\circ} \mathrm{C}$. Next, $2.2 \mathrm{~g}$ of phosphorus trichloride was charged into the flask. The mixture was stirred for 4 hours. After that, the flask was taken out of the oil bath and cooled to room temperature. The mixture in the flask was then transferred to a separatory funnel and allowed to stand overnight. The lower layer was discarded, while the upper layer was collected and kept sealed.

2.3.2. Synthesis of 2-Decyltetradecyl Amidopropyl-N,Ndimethylamine. $0.05 \mathrm{~mol}$ (22.6 g) of the obtained 2-decyltetradecanoyl chloride was charged into a $100-\mathrm{ml}$ three-neck flask. Afterward, $0.2 \mathrm{~mol}$ of $\mathrm{N}, \mathrm{N}$-dimethyl-1,3-propane diamine was dropped into the flask under stirring. The resulting mixture was washed three times with distilled water and dried at $65^{\circ} \mathrm{C}$ overnight.

2.3.3. Synthesis of 2-Decyltetradecyl Amidopropyl-N,Ndimethylamine Oxide. 9.04 of 2-decyltetradecyl amidopropyl- $N, N$-dimethylamine was added in a $100 \mathrm{~mL}$ threeneck flask. Next, $0.5 \mathrm{~g}$ of ethylenediaminetetraacetic acid disodium salt was dissolved in $20 \mathrm{~mL}$ distilled water and poured into the flask. The flask was immersed in an oil bath at $80^{\circ} \mathrm{C}$ with vigorously stirring. Then, $4.55 \mathrm{~g}$ of hydrogen peroxide was added dropwise using a dropping funnel. After the addition, the solution was stirred for 12 hours, followed by the addition of $0.5 \mathrm{~g}$ of sodium sulfite to remove the excess hydrogen peroxide. The obtained solid product was washed three times with petroleum ether and then dried under vacuum.

\section{Results and Discussion}

3.1. IR Spectra. The IR spectra of the synthesized dimethylamine and its oxide are shown in Figure 1, where A and $\mathrm{B}$ represent dimethylamine and its oxide, respectively. It can be seen from Figure 1 that there was an additional absorption appeared at $916.86 \mathrm{~cm}^{-1}$ in $\mathrm{B}$, which was the characteristic band of the dimethylamine oxide. The bands at $3291.65 \mathrm{~cm}^{-1}$ could be assigned to $\mathrm{N}-\mathrm{H}$ stretching vibration absorption, while the bands at $2917.73 \mathrm{~cm}^{-1}$ and $2849.43 \mathrm{~cm}^{-1}$ could be assigned to the unsymmetric stretching vibration absorption of $\mathrm{CH}_{3}$ and asymmetric stretching vibration absorption of $\mathrm{CH}_{2}$. Another band at $1637.25 \mathrm{~cm}^{-1}$ could be identified as stretching vibration of the $\mathrm{C}=\mathrm{O}$. The absorption at $1375.99 \mathrm{~cm}^{-1}$ inferred to $\mathrm{C}-\mathrm{N}$.

3.2. Mass Spectra. The ESI-MS spectrum of the amine-oxide is shown in Figure 2. The ion at $m / z=469$ corresponded to $[\mathrm{M}+\mathrm{H}]^{+}$, while an $\mathrm{m} / z$ value of 937.94 could be identified as $[2 \mathrm{M}+\mathrm{H}]^{+}$.

3.3. Surface Tension and CMC of the Synthesized Surfactant. The curves of surface tension versus log concentration of the synthesized amine-oxide surfactant at $25^{\circ} \mathrm{C}$ are illustrated in Figure 3. The CMC (critical micelle concentration) values of the surfactant were calculated from interception point of the two tangent straight lines at low concentrations [18]. As it can be seen from Figure 3, the surface tension values gradually decreased with increasing concentration of the synthesized surfactant concentration, reaching a minimum surface tension value of $24.7 \mathrm{mN} / \mathrm{m}$ at $298.15 \mathrm{~K}$, while the surfactant had a CMC value of $2.14 \times 10^{-5} \mathrm{~mol} \cdot \mathrm{L}^{-1}$.

3.4. Polymer Selection. In the surfactant-polymer flooding, the addition of polymer can increase water viscosity and decrease the water/oil mobility ratio. Such a change can lead to a better sweep efficiency and more efficient oil displacement in the swept zone [19]. In view of average permeability of the targeted reservoir, which is $146 \mathrm{mD}$, the HPAM with the molecular weight of $1700 \mathrm{MDa}$ was selected based on the field test experiences [20]. Its viscosity-concentration curve is shown in Figure 4. In view of viscosity loss which caused by mechanical shearing and degradation effects of the heat or bacteria, the concentration polymer was set at $1000 \mathrm{mg} / \mathrm{L}[21]$.

3.5. Interfacial Tension of the Surfactant-Polymer System. It is well known in the literature that as the capillary number is increased, residual saturations are decreased [22]. In 


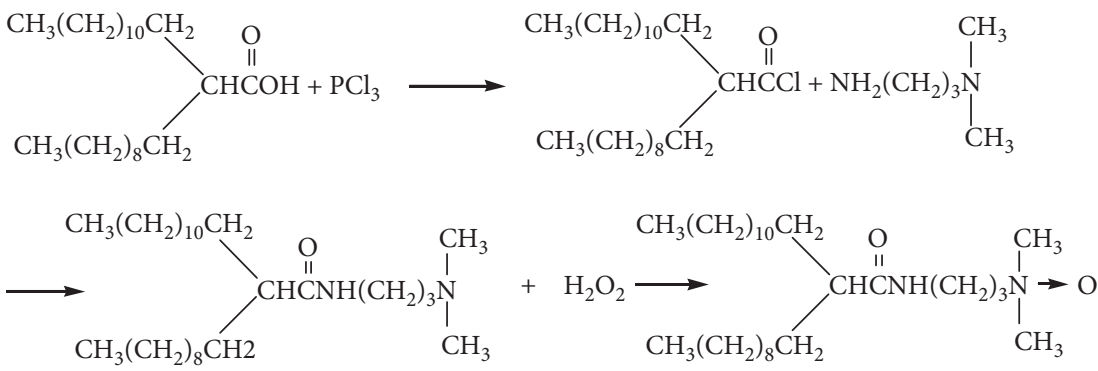

Scheme 1: Synthetic route of the amine-oxide.



FIGURE 1: FT-IR spectrum of the amine and amine-oxide.

theory, based on the definition of the capillary number, the interfacial tension, flow rate, and viscosity of the flooding fluid are associated with the capillary number. However, in the practical flooding process, only the interfacial tension can greatly increase the capillary number. In fact, in a successful displacement process, the injected surfactant slug must achieve ultralow interfacial tension $(<0.01 \mathrm{mN} / \mathrm{m})$ [23].

Factors that affect the interfacial tension include reservoir temperature, salinity, time, and concentration of the surfactant. For a given reservoir, reservoir temperature is fixed. The salinity is relatively stable in most cases. Thus, the effects of surfactant concentration and time on the interfacial tension between crude oil and surfactant-polymer solution were investigated. The surfactant concentration used in the experiments ranged from 0.05 to $0.3 \%$, and the polymer concentration was $1000 \mathrm{mg} / \mathrm{L}$. The results are shown in Figure 5.

As it can be seen from Figure 5, the interfacial tension between surfactant-polymer solution and crude oil could be reduced to ultralow levels in a wide range of surfactant concentrations. In addition, the equilibrium interfacial tension decreased with the increase of the surfactant concentration. The interfacial tension is related to adsorption of the surfactant at the oil/water interface. At a low surfactant concentration, the adsorption rate is faster than that of desorption, so more and more surfactant molecules are accumulated at the oil/water interface, which leads to the reduction of the interfacial tension. When the rate of adsorption equals the rate of desorption, the interfacial tension can reach the lowest value. If the surfactant concentration continues to rise, the monolayer adsorption of the surfactant converts to a bilayer adsorption, and the adsorption rate is slow down while desorption rate is increased, which will result in the increase of the interfacial tension [24].

3.6. Core Flood Experiments. As we know, for reservoirs, the permeability has a big effect on the oil recovery. To simulate reservoir condition, a sand-packed core with a 


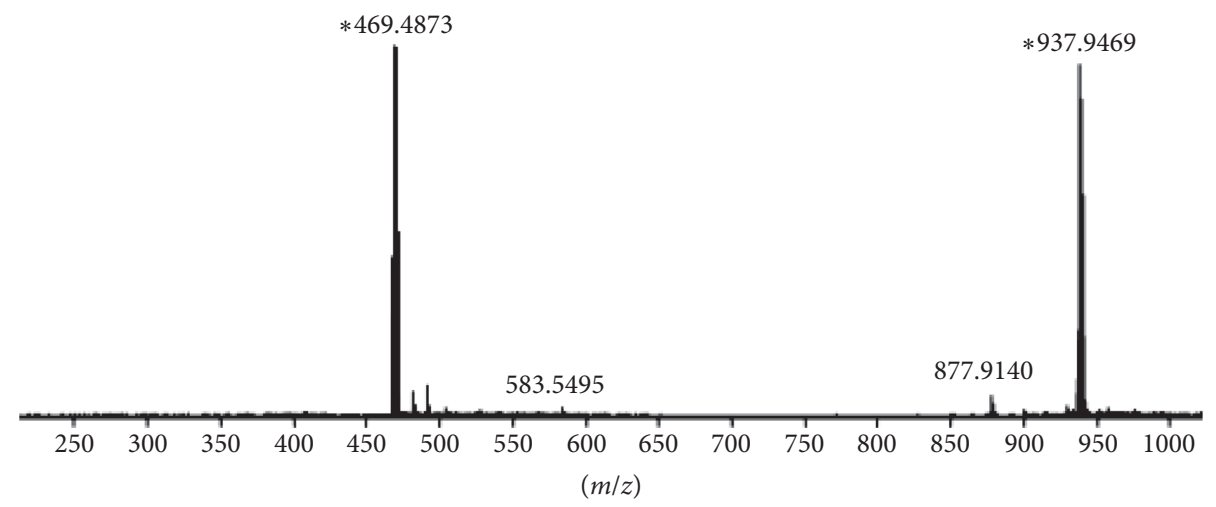

Figure 2: ESI-MS spectrum of the amine-oxide.

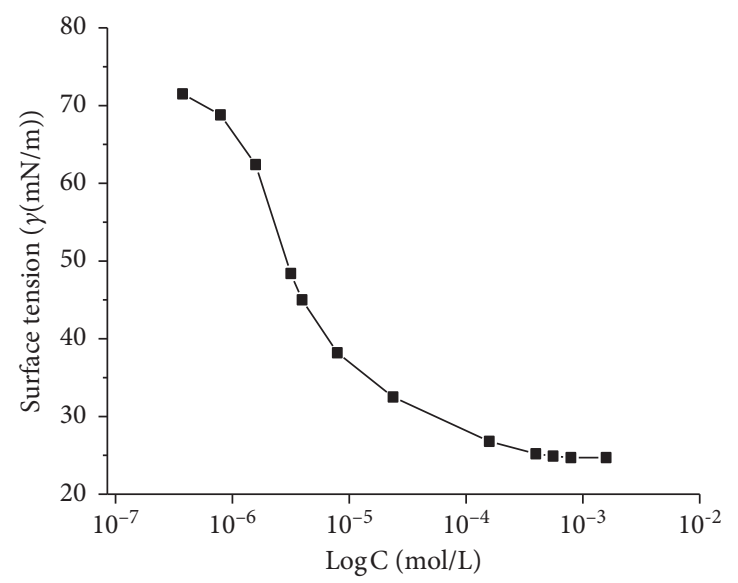

FIgUre 3: The surface tension of the amine-oxide.

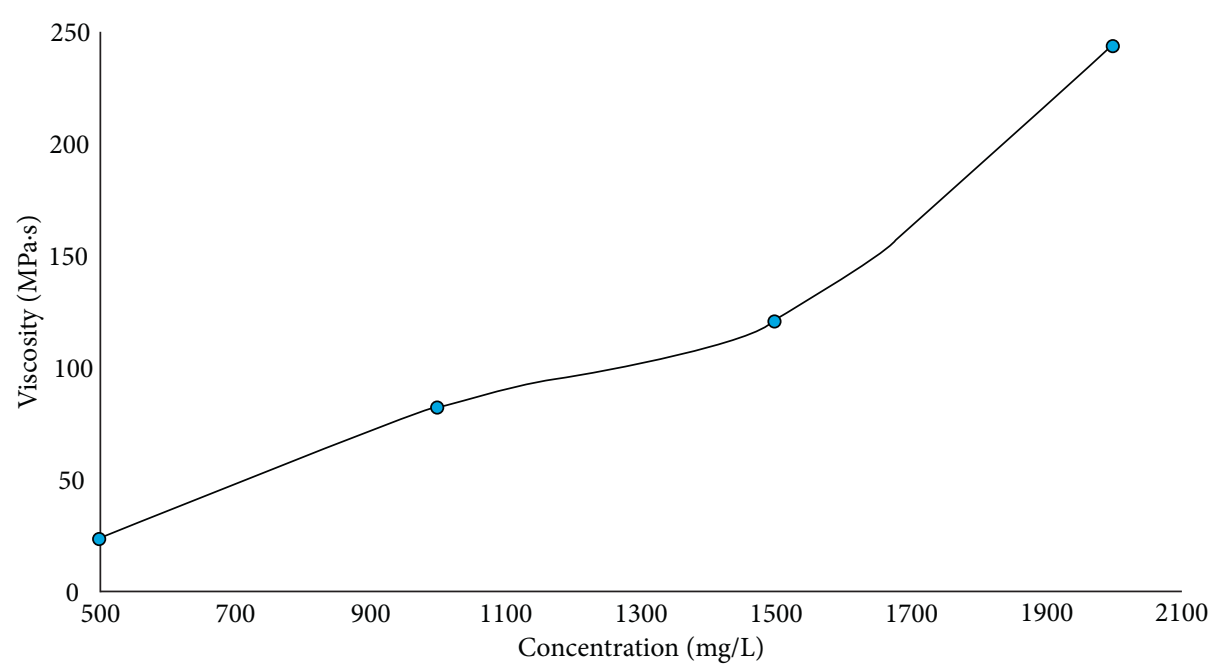

FIGURE 4: Effect of polymer concentration on viscosity.

permeability of $154 \mathrm{mD}$ was used to carry out flooding tests. The core was evacuated for 24 hours and saturated with the formation water. After that, the core was displaced by crude oil until the residual water saturation was created. Then, 0.3 pore volume of the surfactant/polymer slug was injected into the core, followed by water flood using a constant rate of $0.4 \mathrm{ml} / \mathrm{min}$ until $98 \%$ water cut was reached. The results are depicted in Figure 6. It showed a significant increase in the oil production for the surfactant/polymer slug. The water cut dropped after the 


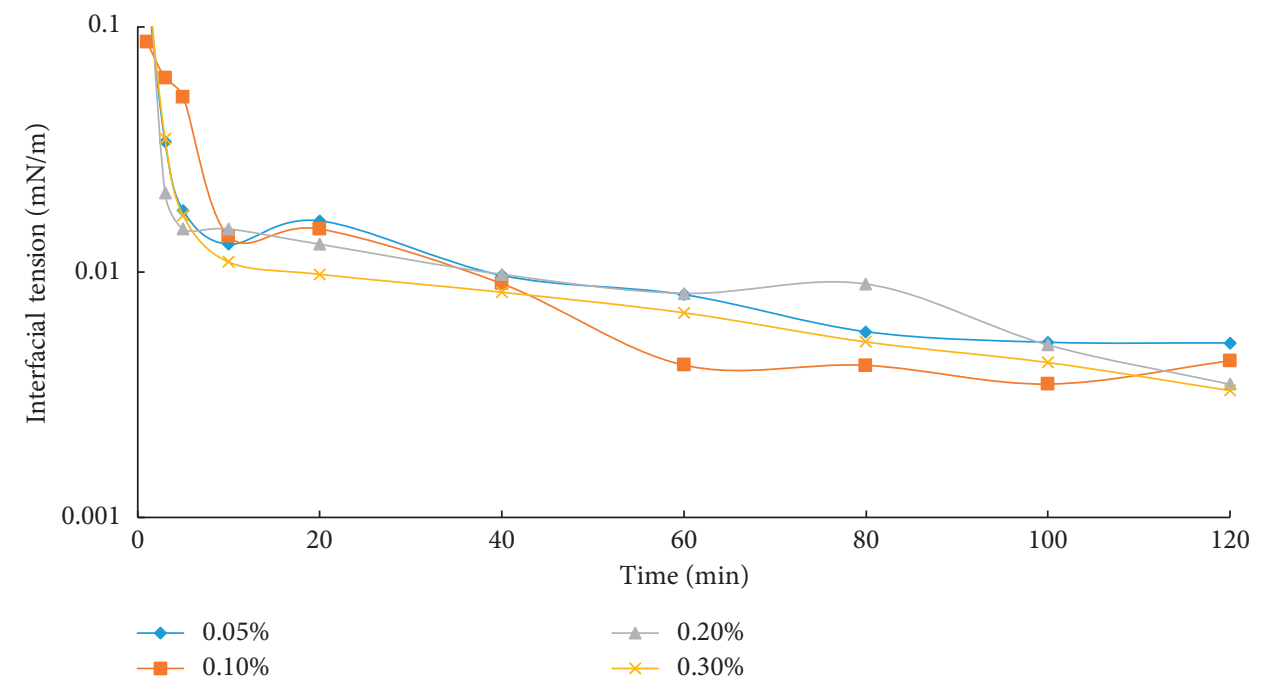

Figure 5: Dynamic interfacial tension of the synthesized surfactant at different concentrations.

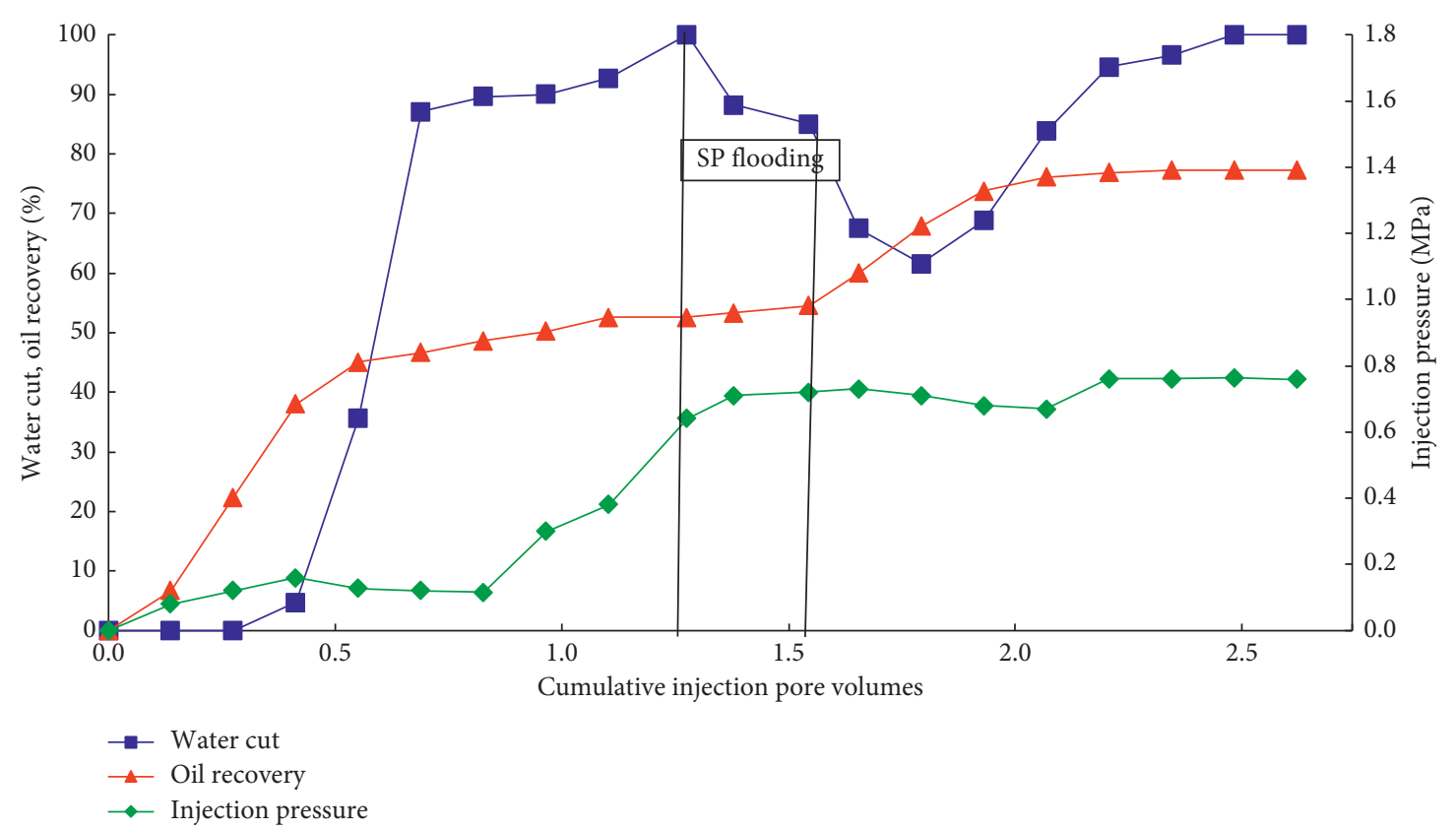

Figure 6: Fluid injected (PV).

injection of the surfactant/polymer slug; in the mean time, the injection pressure rose rapidly. Total oil recovery reached $77.3 \%$, which was 24.7 percent more than water flood.

\section{Conclusion}

Guerbet amine-oxide has been synthesized, and its structure was confirmed by FT-IR and ESI-MS. The surfactant showed a relative lower CMC and interfacial tension. The results obtained from core flood experiment showed that the synthesized surfactant could have the application potential in surfactant-polymer enhanced oil recovery.

\section{Data Availability}

The data used to support the findings of this study are available from the corresponding author upon request.

\section{Conflicts of Interest}

The authors declare that they have no conflicts of interest.

\section{Acknowledgments}

This work was supported by the continuous effective of oil production in Huabei Oil Field Project (grant number 2017E-1507). 


\section{References}

[1] C. Q. Liu and X. F. Jiang, Industry Report of Oil and Natural Gas Development at Home and Abroad, Petroleum Industry Press, Beijing, China, 2016.

[2] H. R. Guo, R. C. Ma, and D. B. Kong, "Success and lessons learned from asp flooding field tests in China," in Proceedings of the SPE/IATMI Asia Pacific Oil \& Gas Conference and Exhibition, Jakarta, Indonesia, October 2017.

[3] P. D. Berger and C. H. Lee, "Improve ASP process using organic alkali," in Proceedings of the SPE/DOE Symposium on Improved Oil Recovery, Tulsa, OK, USA, April 2006.

[4] D. Denney, "Progress and effects of ASP flooding," Journal of Petroleum Technology, vol. 65, no. 1, pp. 77-81, 2013.

[5] Y. Y. Zhu, Y. Zhang, J. L. Niu, W. D. Liu, and Q. F. Hou, "The research progress in the alkali-free surfactant-polymer combination flooding technique," Petroleum Exploration and Development, vol. 39, no. 3, pp. 371-376, 2012.

[6] D. Levitt, A. C. Jackson, C. Heinson et al., "Identification and evaluation of high-performance eor surfactants," in Proceedings of the SPE/DOE Symposium on Improved Oil Recovery, Tulsa, OK, USA, April 2006.

[7] Z. K. Zhao, Z. S. Li, W. H. Qiao, and L. B. Cheng, "Dynamic interfacial behavior between crude oil and octylmethylnaphthalene sulfonate surfactant flooding systems," Colloids and Surfaces A: Physicochemical and Engineering Aspects, vol. 259, no. 1-3, pp. 71-80, 2005.

[8] P. Posocco, A. Perazzo, V. Preziosi, E. Laurini, S. Pricl, and S. Guido, "Interfacial tension of oil/water emulsions with mixed non-ionic surfactants: comparison between experiments and molecular Simulations," RSC Advances, vol. 6, no. 6, pp. 4723-4729, 2016.

[9] Y. Li, Y. Guo, M. Bao, and X. Gao, "Investigation of interfacial and structural properties of $\mathrm{CTAB}$ at the oil/water interface using dissipative particle dynamics simulations," Journal of Colloid and Interface Science, vol. 361, no. 2, pp. 573-580, 2011.

[10] W. Qiao, Y. Cui, Y. Zhu, and H. Cai, "Dynamic interfacial tension behaviors between Guerbet betaine surfactants solution and Daqing crude oil," Fuel, vol. 102, pp. 746-750, 2012.

[11] Y. F. Wu, P. J. Shuler, M. Blanco, Y. C. Tang, and W. A. Goddard, "A study of branched alcohol propoxylate sulfate surfactants for improved oil recovery," in Proceedings of the Annual Technical Conference and Exhibition, Dallas, TX, USA, October 2005.

[12] J. Lu, P. J. Liyanage, S. Solairaj et al., "New surfactant developments for chemical enhanced oil recovery," Journal of Petroleum Science and Engineering, vol. 120, pp. 94-101, 2014.

[13] L. Brinchi, R. Germani, P. Di Profio et al., "Viscoelastic solutions formed by worm-like micelles of amine oxide surfactant," Journal of Colloid and Interface Science, vol. 346, no. 1, pp. 100-106, 2010.

[14] M. T. García, E. Campos, and I. Ribosa, "Biodegradability and ecotoxicity of amine oxide based surfactants," Chemosphere, vol. 69, no. 10, pp. 1574-1578, 2007.

[15] J. C. Lim and D. S. Han, "Synthesis of dialkylamidoamine oxide surfactant and characterization of its dual function of detergency and softness," Colloids and Surfaces A: Physicochemical and Engineering Aspects, vol. 389, no. 1-3, pp. 166-174, 2011.

[16] R. Wang and Y. Li, "Wetting ability in aqueous mixtures of amine oxide with anionic and nonionic surfactants," Tenside Surfactants Detergents, vol. 51, no. 3, pp. 224-228, 2014.
[17] P. Zhang, W. Z. Huang, Z. F. Jia, C. Y. Zhou, M. L. Guo, and Y. F. Wang, "Conformation and adsorption behavior of associative polymer for enhanced oil recovery using single molecule force spectroscopy," Journal of Polymer Research, vol. 21, p. 523, 2014.

[18] P. Singh, A. Srivastava, and R. Kumar, "Synthesis and characterization of nano micelles of poly $(N$-acrylamidohexanoic acid)-b-poly( $N$-vinylcaprolactam) via RAFT process: solubilizing and releasing of hydrophobic molecules," Polymer, vol. 57, pp. 51-61, 2015.

[19] Y. Du and L. Guan, "Field-scale polymer flooding: lessons learned and experiences gained during past 40 years," in Proceedings of the SPE International Petroleum Conference in Mexico, Puebla Pue, Mexico, November 2004.

[20] W. H. Yan, Study on Hydrodynamic Size and Injectivity of Polymer Solution, Chinese Academy of Sciences, Beijing, China, 2015.

[21] X. H. Lv, X. Lei, and R. Z. Chang, "Selection of polymers with heat-resistance and their evaluation," Advances in Fine Petrochemicals, vol. 12, no. 7, pp. 1-4, 2011.

[22] J. J. Sheng, "Preferred calculation formula and buoyancy effect on capillary number," Asia-Pacific Journal of Chemical Engineering, vol. 10, no. 3, pp. 400-410, 2015.

[23] G. Hirasaki, A. Miller, and M. Puerto, "Recent advances in surfactant EOR," SPE Journal, vol. 16, no. 4, pp. 889-907, 2011.

[24] A. Shioi, Y. Sugiura, and R. Nagaoka, "Oscillation of interfacial tension at a liquid/liquid interface composed of di(2ethylhexyl) phosphoric acid and calcium chloride," Langmuir, vol. 16, no. 22, pp. 8383-8389, 2000. 